BRIEF REPORT

\title{
Children's response to a commercial back-up warning device
}

\author{
R E Sapien, J Widman Roux, L Fullerton-Gleason
}

Injury Prevention 2003;9:87-88

See end of article for authors' affiliations

....................

Correspondence to: Dr Robert E Sapien, Department of Emergency Medicine, University of New Mexico Health Science Center, ACC 4th Floor West, Albuquerque, New Mexico 87131, USA rsapien@salud.unm.edu

\begin{abstract}
Objective: To determine preschool children's response to a commercial back-up warning alarm in a mock setting of an automobile backing up.

Setting: Preschool parking lot in Albuquerque, New Mexico, USA.

Methods: With subjects acting as their own controls, 33 preschoolers were asked to walk behind a stationary vehicle twice. The first time, the control, no warning sound was emitted from the vehicle. The second time, the vehicle was placed in reverse gear triggering an alarm. Children's responses were recorded by a hidden video camera. Avoidance behavior by the child was considered a positive response. Location and distance to where the response occurred was measured.

Results: Thirty three children, 38-61 months, were studied. None responded to the alarm with avoidance behavior but 18 looked toward the vehicle or hesitated in their gait.

Conclusion: Although over half of the children acknowledged the warning alarm, the device did not elicit avoidance behavior. Mere acknowledgment of the warning device would not prevent injury.
\end{abstract}

n the United States, pedestrian injuries account for $35 \%-40 \%$ of motor vehicle deaths in children. ${ }^{1}$ Ten percent of children injured as pedestrians die or have long term sequelae such as debilitating head or extremity injury. ${ }^{2-7}$ Child pedestrian injuries occur in traffic or non-traffic settings. Younger children tend to be injured more in non-traffic incidents such as parking lots or driveways. ${ }^{2}$ Vehicles backing up in driveways and parking lots are responsible for the majority of pedestrian injuries involving children younger than age 5. One study found that children are three times at risk for sustaining a non-traffic pedestrian injury if the play area is not separated from the driveway by a fence. ${ }^{8}$ Children from households in which two residences share a driveway have twice the risk of being injured and children from lower socioeconomic levels are at five times the risk of being a driveway pedestrian victim.

Back-up warning devices may be indicated to prevent these injuries but their efficacy has not been measured. Our study was conducted to evaluate the effectiveness of a commercial back-up warning device in a preschool population. We hypothesized that (1) children would be more likely to demonstrate avoidance behavior when a vehicle emitted a warning sound than when it did not; (2) children who responded to the warning device would differ from those who did not respond with respect to age, gender, and development score; and (3) some of those who responded would demonstrate avoidance behavior, for example, by moving away from the vehicle.

\section{METHODS}

This study was a non-randomized comparison in which preschool children, ages 3-5 years, served as their own controls. A local preschool agreed to participate and consent was obtained from the children's parents. Hearing impaired and potentially uncooperative children were excluded. All children were assessed with the Brigance Diagnostic Inventory of Early Development, ${ }^{10}$ a standardized and validated developmental assessment measure administered routinely at the start of the school year. The study was approved by the University of New Mexico Health Sciences Human Subjects Review Board.

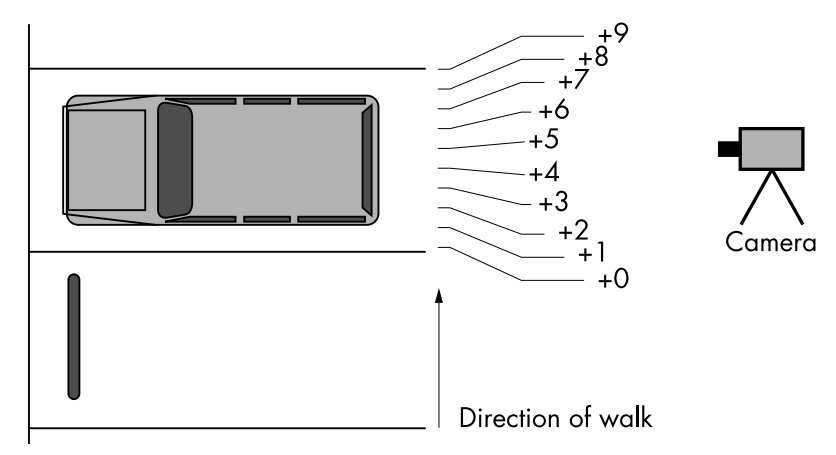

Figure 1 Mock setting of automobile backing up.

A warning device marketed for family cars was installed on a four door automobile. It emitted the same beep heard when a large truck is in reverse gear. This sound was emitted as the child began to walk through the parking lot, behind and perpendicular to the long axis of the vehicle about 18 inches from the rear bumper of the car. This was designated as distance zero (fig l). When the child first walked behind the parked vehicle no sound was emitted (control). Within five minutes, they walked behind the vehicle a second time and the warning device sounded (intervention). A parent or teacher was waiting at the opposite side of the vehicle, but did not coach the children to respond to the warning. The child's behavior was recorded by an unobtrusive VCR camera.

All observations were done in the same parking lot and at the same time of day to control for ambient noise and distractions as best as possible. The children were scored on the distance from zero at which they responded. A positive response was avoidance behavior, for example, stopping or any hesitation in gait. All other actions were considered negative responses. We compared positive and negative responders stratifying by age, gender, distance of response, and Brigance scores.

Sample size calculations were based on the estimate that $10 \%$ of children in the control condition and $50 \%$ in the experimental condition would have a positive response. To achieve a power of $80 \%$, using a type I error rate of $5 \%, 24$ subjects in each condition were required. Logistic regression and 
$\chi^{2}$ analysis were used to evaluate differences in the dichotomous outcome variable ("yes" response to the warning device $v$ "no" response to the device). The response distance was analyzed using analysis of variance. Statistical analyses were performed using JMP (SAS Institutes 1999-2001).

\section{RESULTS}

Thirty three children ages 38-61 months (median 53 months) participated; $57.6 \%$ were male. Brigance scores ranged from $56 \%-100 \%$ but the distribution was negatively skewed, with a median of $84 \%$.

None of the children showed positive responses, that is, avoidance behavior either during the control or experimental phase. For this reason, control data were not further analyzed. Eighteen acknowledged the warning by looking towards the vehicle and hesitating. None of the hypothesized predictor variables (age, gender, Brigance score), however, predicted acknowledgement of the warning. Additionally, inclusion of all three variables in a logistic regression model did not distinguish those who merely acknowledged the warning from the negative responders.

\section{DISCUSSION}

None of the children responded to the warning device positively, that is, with avoidance behavior. Although over half hesitated or otherwise acknowledged the alarm, all would have been injured had this been an actual back-up situation

The results suggest that children who acknowledged the sound were slightly older, had higher Brigance Developmental scores, and tended to be males. Although these differences were not statistically significant, further research is needed to determine whether the failure to find differences merely reflect the sample size.

This study targets the appropriate age group ${ }^{279}$ and injury setting. ${ }^{8}$ Limitations of this study include environmental factors that could not be controlled including ambient noise, weather, and lighting. The study was done over several days and early participants may have talked to others alerting them to what to expect. There may have been selection bias since subjects enrolled may be children of parents who are more safety conscious. If this were the case, however, the bias would be expected to shift the results towards increased positive responses.

\section{Key points}

- As they walked behind a sedan with a commercial back-up warning device, $55 \%$ of children looked toward the vehicle as it emitted the back-up warning sound.

- None of the children responded to this commercial back-up warning device with avoidance behavior.

- A commercial back-up warning device, which emits a warning sound, did not illicit effective injury prevention action in the preschool population studied.

The back-up warning device tested appears inadequate for injury prevention in this population. It may need to be coupled with an educational intervention or may be more effective with older children.

\section{ACKNOWLEDGEMENTS}

We would like to thank the YDI-Headstart Preschool Program in Albuquerque, New Mexico. Funding provided by a medical student research grant, Office of Graduate Medical Studies, University of New Mexico Health Sciences Center.

\section{REFERENCES}

Winn D, Agran P, Castillo D. Pedestrian injuries to children younger than 5 years of age. Pediatrics 1991;88:776-82.

2 Olson L, Sklar D, Cobb, D, et al. Analysis of Childhood Pedestrian Deaths in New Mexico, 1986-1990. Ann Emerg Med 1993:22:512-16

3 Rivara F P. Child pedestrian Injuries in the United States: current status of the problem, potential interventions, and future directions. Am J Dis Child 1990; 144:692-6

4 Partrick D, Bensard D, Moore E, et al. Driveway crush injuries in young children: a highly lethal, devastating, and potentially preventable event. $J$ Pediatr Surg 1998;33:1712-15.

5 Holland AJ, Liang RW, Singh SJ, et al. Driveway motor vehicle injuries in children. Med J Aust 2000;173:503.

6 Nadler EP, Courcoulas AP, Gardner M, et al. Driveway injuries in children: risk factors, morbidity and mortality. Pediatrics 2001:108:326-8

7 Silen ML, Kokoska ER, Fendya DG, et al. Rollover injuries in residential driveways: age-related patterns of injury. Pediatrics 1999;104:7.

8 Agran P, Winn D, Anderson C. Differences in child pedestrian injury events by location. Pediatrics 1994:93:284-8.

9 Robertson L. Car design and risk of pedestrian deaths. Am J Public Health 1990:80:609-10.

10 Brigance AH. Brigance diagnostic inventory of early development. N Bellerica, MA: Curriculum Associates, Inc, 1991. 